\title{
Low intensity peripheral muscle conditioning improves exercise tolerance and breathlessness in COPD
}

\author{
C.J. Clark, L. Cochrane, E. Mackay
}

\begin{abstract}
Low intensity peripheral muscle conditioning improves exercise tolerance and breathlessness in COPD. C.J. Clark, L. Cochrane, E. Mackay. (CERS Journals Ltd 1996. ABSTRACT: This randomized, controlled study investigated the physiological effects of a specially designed 12 week programme of isolated conditioning of peripheral skeletal muscle groups. The programme required minimal infrastructure in order to allow continued rehabilitation at home after familiarization within hospital.

Forty eight patients, aged 40-72 yrs with chronic obstructive pulmonary disease (COPD) (mean (SD) forced expiratory volume in one second (FEV1) 61 (27)\% of predicted normal) were randomly allocated into training $(n=32)$ and control $(n=16)$ groups. Physiological assessments were performed before and after the 12 week study period, and included peripheral muscle endurance and strength, whole body endurance, maximal exercise capacity (maximum oxygen consumption $\left(V^{\prime} \mathrm{O}_{2}\right.$, max $)$ ) and lung function.

The training group showed significant improvement in a variety of measures of upper and lower peripheral muscle performance, with no additional breathlessness. Whole body endurance measured by free arm treadmill walking increased by $6,372(3,932-8,812) \mathrm{J}(\mathrm{p}<0.001)$. Symptom-limited maximal $V^{\prime} \mathrm{O}_{2}$ was unchanged. However, the training group showed a reduction in ventilatory equivalents for oxygen and carbon dioxide, both at peak exercise and at equivalent work rate ( $\left.W_{\max }\right)$.

In summary, low intensity isolated peripheral muscle conditioning is well-tolerated, simple and easy to perform at home. The various physiological benefits should enable patients across the range of severity of chronic obstructive pulmonary disease to improve daily functioning.

Eur Respir J., 1996, 9, 2590-2596.
\end{abstract}

Dept of Respiratory Medicine, Hairmyres Hospital, East Kilbride, Glasgow, Scotland.

Correspondence: C.J. Clark

Dept of Respiratory Medicine

Hairmyres Hospital

East Kilbride

Glasgow

G75 8RG

Scotland

Keywords: Chronic obstructive pulmonary disease

dyspnoea

home care

pulmonary rehabilitation

skeletal muscle

Received: April 41995

Accepted after revision August 281996
Exercise training is a key component of pulmonary rehabilitation programmes. Patients with chronic obstructive pulmonary disease (COPD) have a wide range of exercise tolerance and, therefore, two distinct outcome objectives of training need to be identified. First will it be possible to obtain the physiological benefits arising from improved cardiorespiratory fitness. This is thought to be achievable only if the patient can exercise during each session at sufficient intensity to induce these physiological changes [1]. The second objective arises when incapacitating breathlessness prevents high intensity exercise and, therefore, aerobic training. This consists of strategies designed to improve mobility within the constraints of unaltered cardiorespiratory performance.

To address the needs of all of these various groups of patients, a range of exercise programmes has recently been suggested [2]. This randomized, controlled study investigated the physiological effects of one of these, a specially designed programme of isolated conditioning of peripheral skeletal muscle groups. The programme was designed to minimize infrastructure requirements in order to allow performance at home after familiarization. The principle was to use frequent repetitions on unloaded muscle groups in order that each individual exercise would be tolerated in isolation, therefore allowing progression through a cumulative set of exercises performed sequentially, without debilitating breathlessness. The study design included a control group, against which to judge the magnitude of physiological changes induced in the training group, and also to give insight into the reproducibility of these measurements of muscle performance with time in COPD patients, which is important if they are to be used in evaluation of rehabilitation outcome.

\section{Methods}

\section{Patients}

Forty eight patients were selected from the hospital chest clinic. They were all suffering from COPD as defined by the American Thoracic Society [3]. The minimum treatment consisted of inhaled bronchodilator and inhaled steroid; the maximum treatment included nebulized bronchodilators and long-term oral steroids.

\section{Study design}

The 48 patients were randomly allocated into training $(n=32)$ or control $(n=16)$ groups, with a $2: 1$ training 
versus control ratio. Measurements of peripheral muscle endurance and strength, whole body endurance, maximal exercise capacity (maximal oxygen consumption $\left(V^{\prime} \mathrm{O}_{2}\right.$,max $)$ ) and lung function were made in both groups before and after the 12 week study period. Patients in the training group attended the hospital once a week to perform the Hairmyres Home Exercise Programme (table 1) under the supervision of the physiotherapist. They were instructed to carry out the programme every day at home and to keep a record of their performance in a training log. Initially, patients undertook each exercise for $30 \mathrm{~s}$. This was progressively increased to 45 and then $60 \mathrm{~s}$ if the optimal number of repetitions and a low rating for breathlessness (modified Borg Score) were achieved. Patients in the control group were asked to continue with their usual daily routine during the study period.

\section{Peripheral muscle endurance and strength measure- ments}

Isotonic muscle endurance (with external loading). The Westminster Pulley system was used to assess isotonic muscle endurance of the upper and lower limbs, with external weight loading of up to 5 and $112 \mathrm{~kg}$, respectively. The time taken to fatigue and the total number of repetitions during this time were recorded. (Details of the testing procedures are given in the Appendix).

Isotonic muscle endurance (without external loading). The series of 10 isolated sequential isotonic exercises, which were developed and used as the basis of the training during the 12 week study period (table 1), also served as an evaluation ("The 30 Second Exercise Evaluation") before and after the study period both in the control patients and those undergoing training. For the purposes of familiarization, each exercise was demonstrated by the therapist and then copied by the patient. The patient was then instructed to carry out each exercise in sequence for $30 \mathrm{~s}$ (rest periods were permitted during the $30 \mathrm{~s}$ ). The modified Borg score for breathlessness and the number of repetitions were recorded at the end of each $30 \mathrm{~s}$ exercise period. There was a recovery period between each exercise to allow the patient's breathlessness score to return to zero (approximately $1-5 \mathrm{~min})$.

Isokinetic muscle strength. A subgroup of patients $(\mathrm{n}=10)$ from both groups underwent muscle strength assessment using the Kin-Com. The Kin-Com 500-H (Chattanooga Group Inc., Chattanooga, Tenessee, USA) is a hydraulically-driven, microcomputer-controlled device, which was used to assess isokinetic muscle strength of the upper and lower limbs. (Details of the testing procedures are given in the Appendix).

\section{Whole body endurance measurements}

Endurance walk test. The endurance walk test was performed on a treadmill (Powerjog E10, Sport Engineering Ltd, Birmingham, UK). A speed of $1.5-3.5 \mathrm{~km} \cdot \mathrm{h}^{-1}$ was selected, depending on the sex, age and anthropometric
Table 1. - The Hairmyres home exercise programme

1. Shoulder circling

a) Forwards - circle shoulder girdle forwards.

b) Backwards - circle shoulder girdle backwards.

2. Full arm circling (Relaxation encouraged throughout)

a) Right arm - passing arm as near as possible to side of head, circle arm in a large circle.

b) Left arm - repeat with left arm.

3. Increasing and decreasing circles

a) Right arm - extend arm out to the side at shoulder height. Progressively increase size of circle for a count of 5 , then decrease to a count of 5 .

b) Left arm - repeat with left arm.

4. Abdominal exercise

Sit in chair. Tighten abdominal muscles, hold for count of 4 . Return to starting position.

5. Wall press-ups

Stand with feet should-width apart and a comfortable distance from the wall. Place hands on wall shoulder-width apart and

6. Sitting to standing bend at elbow then push arms straight again.

7. Quadriceps exercise

Use a dining room chair, from a sitting position, stand up then sit down again.

a) Right leg - Sit in a chair and straighten right knee. Tense thigh muscle and hold for count of

b) Left leg - repeat with left leg.

8. Calf raises Go up on to toes, raising heels off the floor then return to starting position.

9. Calf alternates/walking on the spot

Allow one knee to bend, keeping toes on the ground. Bend other knee, whilst straightening first knee. Repeat this bending/straightening of knees, i.e. walking on the spot.

10. Step-up Step-up with right foot on to step then bring up left foot. Step down with right foot then with left foot.

characteristics of the individual patient. The patient walked at his or her selected speed until exhaustion. If this did not occur within 5 min (prestudy assessment) and within $10 \mathrm{~min}$ (poststudy assessment), the speed was progressively increased by $1 \mathrm{~km} \cdot \mathrm{h}^{-1}$ every minute until exhaustion. The total time and distance walked were recorded at the end of the test.

The total work performed during the endurance walk test was calculated in three stages. The power (watts) at each speed was determined using the following equation:

$$
\text { Power }(\mathrm{W})=\underset{\left(\mathrm{km} \cdot \mathrm{h}^{-1}\right) \times 0.1635}{0.536 \times \text { weight }(\mathrm{kg})} \times 0.625 \times \text { speed }
$$

The work (joules) performed at each speed was determined using the following equation:

$$
\text { Work }(\mathrm{J})=\text { power }(\mathrm{W}) \times \text { time }(\mathrm{s})
$$

The total work performed was a summation of the work performed at each speed.

Aerobic exercise testing. Progressive incremental exercise was undertaken by the patients 10 min after bronchodilator administration. Exercise was performed on 
an electronically-braked ergometer (Corival 300 Gould Inc., Medical Products Division, Dayton, Ohio, USA), in which the workload was increased by 5-20 W increments depending on the sex, age, anthropometric characteristics and baseline lung function measurements. The same individualized protocol was used for poststudy evaluation. Work increments were increased at 1 min intervals, while the subject was pedalling at a frequency of 40-60 cycles. $\mathrm{min}^{-1}$, until he or she was exhausted (System 2900, Sensor Medics BV, Anaheim, California, USA) [5].

Heart rate was measured with a 3 -lead C\&W diascope. Minute ventilation $\left(V^{\prime} \mathrm{E}\right)$, tidal volume $(V \mathrm{~T})$, and respiratory frequency $\left(f^{\prime} \mathrm{R}\right)$ were measured by means of a mass flow meter. Volume measurements were corrected to body temperature and pressure saturated with water vapour (BTPS units). The oxygen consumption $\left(V^{\prime} \mathrm{O}_{2}\right)$ and carbon dioxide production $\left(V^{\prime} \mathrm{CO}_{2}\right)$ were calculated from the fractional concentrations of oxygen and carbon dioxide in the mixed expired gas.

An indication of respiratory reserve capacity was obtained by expressing the maximum exercise ventilation as a percentage of the maximum voluntary ventilation (MVV) (postbronchodilator forced expiratory volume in one second $(\mathrm{FEV} 1) \times 35)$ [6]. Maximum cardiac frequency $\left(f^{\prime} \mathrm{C}\right.$,max $)$ was predicted from the formula: $f^{\prime} \mathrm{C}$, max $=210-0.65 \times$ age $(y r s)$ [7]. Oxygen pulse was defined as oxygen consumption per heart beat $\left(\mathrm{mL} \cdot\right.$ beat $\left.^{-1}\right)$.

Arterial oxygen and carbon dioxide tension $\left(\mathrm{Pa}_{\mathrm{a}} \mathrm{O}_{2}\right.$ and $P$ a, $\mathrm{CO}_{2}$, respectively) were monitored throughout exercise with a transcutaneous electrode (TINA TCM3, Radiometer Ltd, Radiometer Copenhagen, Crawley, UK), after in vivo calibration using a single arterial blood sample obtained from the radial artery [8]. During submaximal exercise and at peak exercise, the patients were instructed to estimate their sense of breathlessness according to a modified Borg Score [9].

\section{Lung function assessment}

Baseline spirometry and flow volume analysis were performed with a dry rolling seal spirometer (system 5000 IV; Gould Electronics, Gould Inc., Medical Products Division, Dayton, Ohio, USA). Total lung capacity and residual volume were measured by helium dilution. The diffusing capacity was measured using the singlebreath method. Dynamic spirometry was repeated following administration of salbutamol $(5 \mathrm{mg}$ in $1 \mathrm{~mL})$ via a Wright mini-nebulizer.

\section{Statistical analysis}

The distributions of data sets were viewed for normality using frequency plots. If considered to deviate from a normal distribution a nonparametric statistical test was used. Formal statistical analysis examined changes from baseline both in the training and control groups, i.e. to reduce "between-subject" variability by looking at only "within-subject" variability, using either Student's t-test (parametric) or Mann-Whitney U-test (nonparametric). To illustrate the magnitude of the effects of "treatment", examples of key efficacy variables are given in "before and after" plots with summaries of mean differences and 95\% confidence intervals $(95 \%$
CI). Where a nonparametric test has been used, the median plus the interquartile range (IQR) are presented. For clarity, not all the data has been presented in these plots but, where relevant, summary statistics are given for other efficacy variables within the text.

\section{Results}

Table 2 provides a summary of the key baseline characteristics for the training and control group. The groups were generally well-matched at the outset of the study. Formal statistical analysis between the two groups showed no significant difference in these key characteristics.

\section{Peripheral muscle endurance and strength}

Isotonic muscle endurance (with external loading). After the study period, the training group showed a significant increase in the number of repetitions performed for lower body exercise (fig. 1). There were similar findings

Table 2. - Key characteristics of patients at outset of study

\begin{tabular}{lcc}
\hline & Training & Control \\
\hline Age yrs & $58 \pm 8$ & $55 \pm 8$ \\
BMI kg.m & $24 \pm 3$ & $27 \pm 5$ \\
FEV1 L & $1.72 \pm 0.83$ & $1.44 \pm 0.59$ \\
$\%$ pred & $64 \pm 29$ & $55 \pm 22$ \\
$\mathrm{RV} \mathrm{L}$ & $2.84 \pm 1.18$ & $2.46 \pm 0.85$ \\
$\%$ pred & $141 \pm 45$ & $129 \pm 28$ \\
$P_{\mathrm{a}, \mathrm{O}_{2}} \mathrm{kPa}$ & $10.1 \pm 1.7$ & $9.9 \pm 1.0$ \\
$V^{\prime} \mathrm{O}_{2} /$ weight $\mathrm{mL} \cdot \mathrm{s}^{-1} \cdot \mathrm{kg}^{-1}$ & $17.0 \pm 5.5$ & $14.7 \pm 4.2$ \\
$f^{\prime} \mathrm{C}, \max \% \mathrm{pred}$ & $75 \pm 11$ & $72 \pm 11$ \\
$V^{\prime} \mathrm{E}$,max L$\cdot \mathrm{min}^{-1}$ & $38.5 \pm 14.7$ & $37.2 \pm 13.6$ \\
\hline
\end{tabular}

Values are presented as mean \pm SD. BMI: body mass index; FEV1: forced expiratory volume in one second; $\%$ pred: percentage of predicted value; RV: residual volume; $P \mathrm{a}, \mathrm{O}_{2}$ : arterial oxygen tension; $V^{\prime} \mathrm{O}_{2}$ : oxygen consumption; $f^{\prime} \mathrm{C}$,max: maximal cardiac frequency; $V^{\prime} \mathrm{E}$,max: maximal minute ventilation.
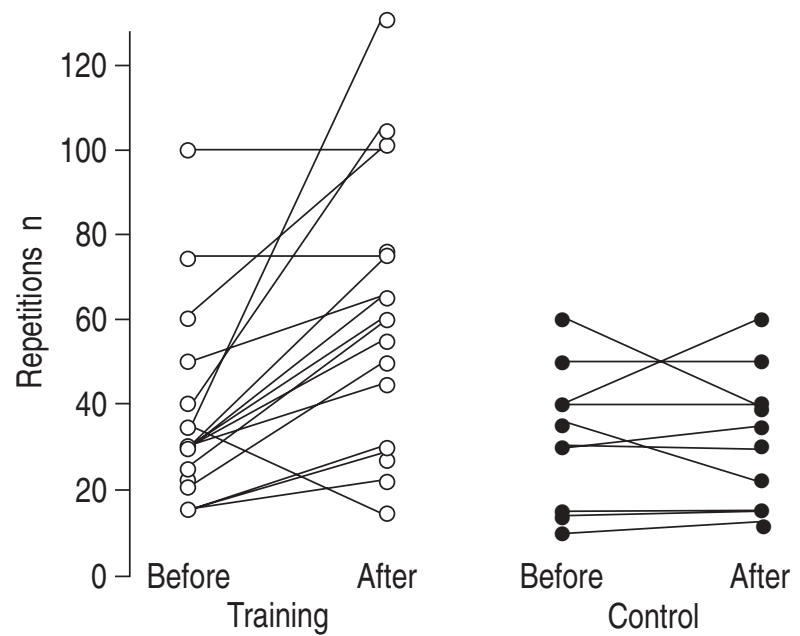

Fig. 1. - A comparison of isotonic lower body muscle endurance with external loading (i.e. knee extension and flexion) in the training $\mathrm{O}-\mathrm{O}$ and control $\longrightarrow$ groups, before and after the study period. The mean difference in repetitions for the training group was 25 (95\% CI 13-37) versus 1 (95\% CI -5-7) for the control group ( $\mathrm{p}<0.001$, by Student's t-test). $95 \%$ CI: $95 \%$ confidence interval. 
for upper body exercise, i.e. shoulder flexion (mean difference $=12(95 \%$ CI 8-15); $\mathrm{p}<0.001$, using Student's $\mathrm{t}$-test). The results were comparable using nonparametric test, i.e. Mann Whitney U-test (median difference = 12 (IQR 6-16); $\mathrm{p}<0.001$ ). The control group showed little change from baseline both for knee extension (mean difference $=1(95 \%$ CI 5-7)) and shoulder flexion (mean difference $=2(95 \%$ CI 0-4) $)$. The total time taken to fatigue did not change significantly in either group.

Isotonic muscle endurance (without external loading the 30 Second Exercise Evaluation). After the 12 week study period, those in the training group showed a highly significant increase $(\mathrm{p}<0.001)$ in the number of repetitions performed during the $30 \mathrm{~s}$ exercise periods both in upper and lower body exercises. Two examples are given in figure 2 (shoulder circling) and figure 3 (sit to stand). Results from the other upper and lower body

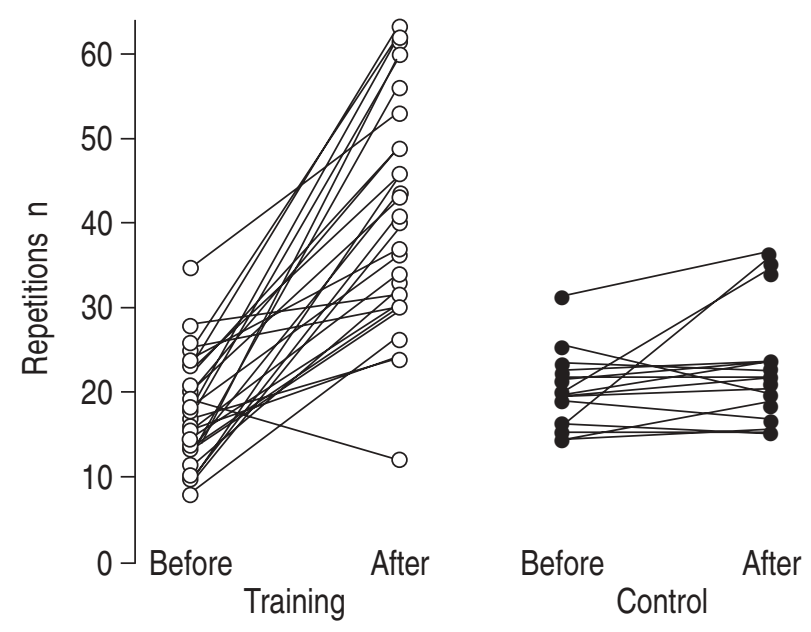

Fig. 2. - A comparison of isotonic upper body muscle endurance without external loading (i.e. shoulder circling) in the training $\mathrm{O}-\mathrm{O}$ and control $\longrightarrow$ groups, before and after the study period. The mean difference in repetitions for the training group was 24 (95\% CI 19-28) versus $2(95 \%$ CI $-1-5)$ for the control group $(\mathrm{p}<0.001$, by Student's t-test). $95 \%$ CI: $95 \%$ confidence interval.

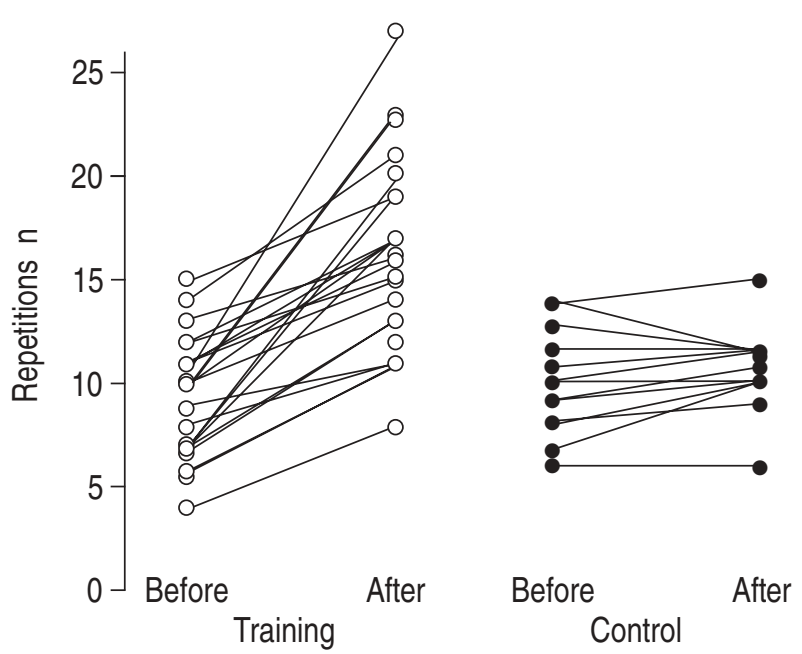

Fig. 3. - A comparison of isotonic lower body muscle endurance without external loading (i.e. sit to stand) in the staining $\mathrm{O}-\mathrm{O}$ and control - groups, before and after the study period. The mean difference in repetitions for the training group was 6 (95\% CI 5-7) versus $1(95 \%$ CI $0-1)$ for the control group $(\mathrm{p}<0.001$, by Student's t-test). $95 \%$ CI: $95 \%$ confidence interval. exercises showed a similar magnitude of change, e.g. press-ups (training group: mean difference $=9(95 \%$ CI 7-10) versus control group: mean difference $=1$ (95\% CI 1-2); $\mathrm{p}<0.001)$. Nonparametric testing revealed similar results in all the exercises, e.g. step-up (training group: median difference $=5$ (IQR 3-8) versus control group: median difference $=0$ (IQR 2-3); $\mathrm{p}<0.001)$. There was no significant change in Borg scores for breathlessness after performing each exercise in either group, after the study period.

Isokinetic Muscle Strength (Kin-Com). There was no change in either group after the study period. For brevity this data is not presented.

\section{Whole body endurance}

Endurance walk test. Figure 4 shows that there was a highly significant change in the endurance work $(J)$ performed by the training group after the study period. The control group also increased their endurance work, but a comparison of the mean change for each group shows a notable difference (control group: mean difference $=430(95 \%$ CI 36-824) versus (training group: mean difference $=6,372(95 \%$ CI 3,932-8,812); $<<0.001)$.

Aerobic exercise testing. Essentially, there was no significant difference in either the training group or the control group in cardiorespiratory performance at peak exercise or at the same workload after training (i.e. the $W \max$ determined on the pretraining evaluation). The training group showed a reduction in ventilatory equivalents for oxygen $\left(V^{\prime} \mathrm{E}, \mathrm{O}_{2}\right)$ and carbon dioxide $\left(V^{\prime} \mathrm{E}, \mathrm{CO}_{2}\right)$ at peak exercise (mean difference $=-3.75(95 \% \mathrm{CI}$ 6.42 to -1.08$), \mathrm{p}=0.009$; and mean difference $=-4.08$ ( $95 \%$ CI -6.59 to -1.57$), \mathrm{p}=0.011$, respectively). No such change was seen in the control subjects. Similar trends and levels of statistical significance were seen for $V^{\prime} \mathrm{E}, \mathrm{O}_{2}$ and $V^{\prime} \mathrm{E}, \mathrm{CO}_{2}$ at equivalent work rates $\left(W_{\max }\right)$, and the

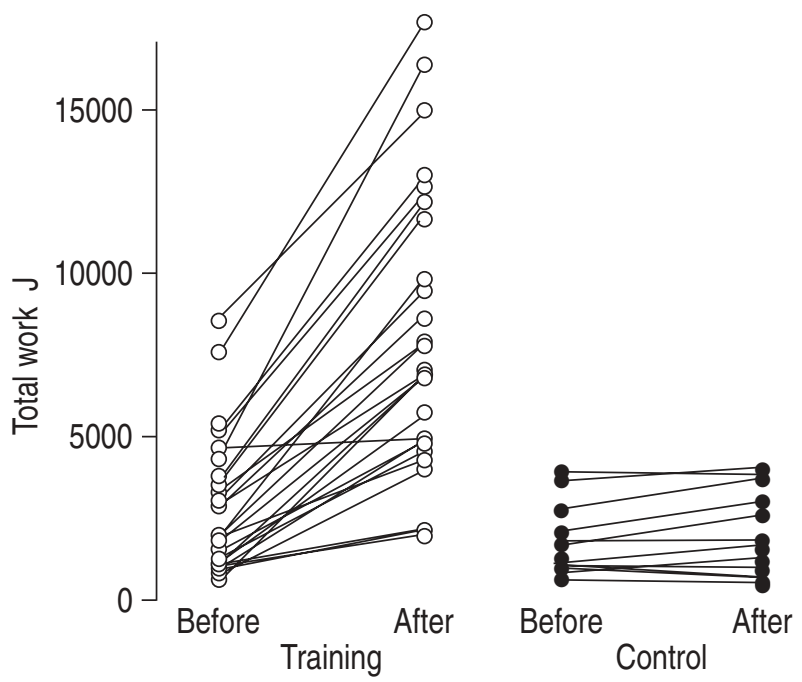

Fig. 4. - A comparison of total work performed on the treadmill (endurance walk test) in the training $\mathrm{O}-\mathrm{O}$ and control $\bullet-$ groups, before and after the study period. The mean difference for the training group was $6,372 \mathrm{~J}(95 \% \mathrm{CI} 3,932-8,812)$ versus $430 \mathrm{~J}$ (95\% CI $36-824$ ) for the control group ( $\mathrm{p}<0.001$, by Student's t-test). 95\% CI: $95 \%$ confidence interval. 
results of nonparametric analysis were still in keeping with these trends $\left(V^{\prime} \mathrm{E}, \mathrm{O}_{2} \mathrm{p}=0.032\right.$ and $V^{\prime} \mathrm{E}, \mathrm{CO}_{2} \mathrm{p}=0.008$ using Mann Whitney U-test): only four of the 48 patients showed oxygen desaturation (three of the training subjects, and one control subject). An analysis of the patients' reasons for stopping exercise on their prestudy evaluation showed that the majority stopped because of dyspnoea $(40 \%)$. In addition, a substantial number stopped because of leg fatigue (21\%) and a further $19 \%$ stopped because of a combination of leg fatigue and shortness of breath. The remaining patients failed to identify a specific reason and cited "general fatigue".

\section{Lung function}

After training, there was no significant change from baseline in FEV1 (L) in either group. There was an increase in the residual volume in the control group (median difference $=0.330$ (IQR 0.05-0.49); $\mathrm{p}=0.02$ using Mann Whitney U-test).

\section{Discussion}

High intensity whole-body exercise programmes suitable for improving fitness in normal subjects are very often not tolerated by COPD patients because of reduced ventilatory reserve and incapacitating breathlessness [10]. Nevertheless, improvements in mobility have been reported [11] following a variety of pulmonary rehabilitation programmes, mainly in uncontrolled studies. One explanation of such improvements may be the effects of regular exercise in countering the "vicious cycle" of deconditioning [12], which can contribute to exercise intolerance through leg fatigue for example.

This randomized, controlled study investigated the physiological benefits of an exercise programme designed for rehabilitation of COPD patients and concentrating on isolated conditioning of peripheral skeletal muscles rather than whole-body aerobic training, on the premise that a cumulative set of individual limb exercises should be better tolerated by the patients than whole body exercise and might improve mobility despite unaltered disease severity. Commonly used exercises, such as press-ups and abdominal muscle contractions, were adapted to take into account the likelihood that the patients might experience inordinate breathlessness, for example when lying horizontally. The programme also incorporated a number of upper limb girdle exercises designed to improve endurance of these particular muscle groups, which act as accessories to respiration [13]. We concentrated on isotonic exercises with no extra external weight loading and, therefore, no requirements for gymnasium equipment.

There are other home exercise rehabilitation programmes for COPD patients, which have been comprehensively reviewed by CASABURI [12], but these rely mainly on walking or other whole-body exercise, such as stairclimbing. In the two studies which did include exercise of smaller muscle groups $[14,15]$, the authors did not perform specific measures of peripheral muscle strength or endurance, so we are unable to make comparisons with our own study. Whole body performance showed little improvement. Ten out of the 15 studies quoted by CASABURI [12] failed to incorporate a control group. This is essential in rehabilitation, where measurable physiological benefits tend to be small and have to take account of the natural variability and potential deterioration of the condition.

The training group showed major improvements, not only in endurance of individual muscles but also in whole-body exercise (i.e. treadmill walking), despite a lack of change in maximal cardiorespiratory fitness [10]. It is also interesting to note the magnitude of the training effect through the increase in number of each of the isotonic muscle repetitions achievable in $30 \mathrm{~s}$, compared with the controls who showed very reproducible results on repeat testing for this and every other aspect of muscle and exercise evaluation. Since this particular assessment is essentially of the speed of repeated exercises, we would speculate that the improvements seen may include a component of improved neuromuscular coupling response time, as has been reported in normal subjects following similar muscle training [16]. This, like endurance, is of direct relevance to daily tasks which require optimal "global" locomotor function. It should also be noted that this increased $30 \mathrm{~s}$ activity was not at the cost of any increase in breathlessness. Furthermore, the programme was well-tolerated by patients, i.e. not associated with severe or uncomfortable levels of breathlessness, as judged by the direct (Borg) measurements which were made during exercise.

The benefits noted can be ascribed to the programme and not to reduction in disease severity, as can be seen by the lack of significant lung function change. The likely mechanism by which improved whole-body endurance exercise can be achieved requires brief consideration. Of the two possible physiological mechanisms [17], an increase both in stroke volume and efficiency of peripheral oxygen extraction by skeletal muscle, the latter is the most likely outcome of the present programme in view of the improvements seen in the isotonic endurance test (Westminster), the $30 \mathrm{~s}$ isotonic repetition test and the treadmill endurance test, with no reduction in heart rate or increase in oxygen pulse at equivalent work intensity.

There were two unexpected observations in these evaluations, which merit further study. Firstly, we did not hypothesize that respiratory function would change, but it was interesting that during the 12 week study period the control group showed an increase in hyperinflation (residual volume) not seen in the training group. The time-scale is rather short for disease progression, but it is possible that the physiotherapist's instruction on controlled breathing techniques during the various exercises improved trainers' breathing technique in preventing dynamic airways narrowing during normal ambient breathing, as has been suggested after breathing retraining [18]. There were no differences in drug treatment in the training or control groups which would explain these findings. Further studies should consider looking at the effects of exercise on hyperinflation.

Secondly, the training group showed a reduction in ventilatory equivalents for oxygen $\left(V^{\prime} \mathrm{E}, \mathrm{O}_{2}\right)$ and carbon dioxide $\left(V^{\prime} \mathrm{E}, \mathrm{CO}_{2}\right)$ following the study period. This has been reported following a high intensity training programme [19], i.e. aerobic training at or above the anaerobic threshold [20]. This was an unexpected observation 
in the present low intensity isolated muscle training programme. The reductions are likely to be due to increased efficiency of peripheral muscle oxygen extraction after muscle training, with less contribution from anaerobic (lactic acid) metabolism during exercise [21]. We cannot completely exclude the alternative explanation that lung mechanics may have improved, i.e. reduced physiological dead space/tidal volume $(V \mathrm{D} / V \mathrm{~T})$ ratios, as we noted a trend towards reduction in the training group but this failed to reach statistical significance. We, therefore, performed a further analysis, which showed no correlation between change in ventilatory equivalents and reduction in $V \mathrm{D} / V \mathrm{~T}$ ratios. Regardless of cause, there is a potential benefit in reducing exercise ventilation and, hence, breathlessness in patients with lung disease, especially using a low intensity programme which is applicable across a wide spectrum of disease severity.

It could be argued that our patients had a similar degree of airflow obstruction to those of CASABURI [19] (FEV1 $1.63 \mathrm{~L}$ (61\% pred) and $1.7-1.8 \mathrm{~L}$ (56\% pred), respectively), and could, therefore, have been expected to participate in a higher intensity programme. However, a comparison of cardiorespiratory fitness shows a much poorer level of exercise performance in our patients $\left(V^{\prime} \mathrm{O}_{2}\right.$, max $1.1 \mathrm{~L} \cdot \mathrm{min}^{-1}(57 \%$ pred) versus $1.4-1.6$ $\mathrm{L} \cdot \mathrm{min}^{-1}\left(73 \%\right.$ pred), and $f^{\prime} \mathrm{C}, \max 122$ beats $\cdot \mathrm{min}^{-1}(75 \%$ predicted maximum) versus $145-158$ beats $\cdot \mathrm{min}^{-1}$ (approximately $90 \%$ predicted maximum). Our patients may have the potential to take part in a higher intensity training programme, but, in subjects who are deconditioned and unfamiliar with exercise, it makes sense to introduce them to exercise at a tolerable and realistic level and, thereafter, gradually increase the intensity.

Our study is a "first phase" investigation to evaluate the ability of a new exercise rehabilitation treatment to induce physiological changes designed to enable the patient to improve daily functioning. The next phase is to apply the physiological gains attained and evaluate the benefits achieved in "predetermined goals" for daily functioning, i.e. activities of daily living. It is clear to us, that many of our patients spontaneously translated the 3 month study period's experience into increased activities, such as gardening, shopping etc., such information usually being volunteered enthusiastically as the programme progressed. Furthermore, the virtually unanimous desire of programme "graduates" to continue to use the programme at home on completion of this study strongly suggests that from the patients' perspective the programme was of significant clinical relevance.

In summary, isolated peripheral muscle conditioning is well-tolerated, simple and easy to perform at home. Practical guidance as to how to implement such a programme is already available [2]. The physiological responses during the programme indicate no undue cardiorespiratory stress, and benefits include not only increased endurance both of specific muscle activities and submaximal work, such as walking, but also reduced ventilation and breathlessness during exertion. These are important functions of daily living and, therefore, key targets for training in those patients with chronic obstructive pulmonary disease, for whom high body intensity training may not be possible or may not be appropriate during the early stages of training.

\section{Appendix}

\section{Measurement of muscle endurance and strength}

Westminster Pulley. Upper limb strength was assessed with the patient seated in a chair in the front of the Westminster Pulley with their back to the pulley. Gripping the handle held at shoulder height, the patient repeatedly extended their arm in front then returned the handle to the starting position. The pulley axis was always positioned at $30 \mathrm{~cm}$ from the floor. The weight used was selected according to the patient's height, weight and physique. The minimum weight was the pulley system unloaded and the maximum weight was 5 $\mathrm{kg}$.

Lower limb strength was assessed using knee extension. The patient was seated on a table in front of the pulley system with their back to the pulley. The pulley was positioned parallel with the patient's ankle secured to the pulley rope by a cross-over strap. The patient repeatedly extended and flexed their knee from $90^{\circ}$ of knee flexion to full extension $\left(0^{\circ}\right)$. As for the upper limb assessment, the weight was selected according to the patient's height, weight and physique. The minimum weight was $0.5 \mathrm{~kg}$ and maximum was 112.5 $\mathrm{kg}$.

Patients performed upper and lower assessments in sequence, with a rest period in between to allow full recovery. They were instructed to continue each exercise until they were exhaused. The fatigue time and the total number of repetitions during this time were recorded for each exercise.

Isokinetic muscle strength (Kin-Com 500H). The patient resists the computer controlled movement of the lever arm.

The lower limb test was performed with the patient seated to localize knee movement. Knee extension was tested starting at an angle of $80^{\circ}$ and stopping at an angle of $10^{\circ}$. The start forces used were recognized for knee testing i.e. $50 \mathrm{~N}$ back and forth, $20 \mathrm{~N}$ minimum force and acceleration and deceleration set at "medium". Three isokinetic speeds were used: 30, 60 and 90 degrees $\cdot \mathrm{s}^{-1}$. Following warm-up and familiarization the "overlay" test was performed. Isolated concentric and eccentric movement was repeated 3 times and the mean peak force for the three movements at each of the three speeds was recorded.

The upper limb test was performed with the patient seated and their shoulder joint aligned with the dynamometer head. The force pad was positioned distal to the elbow joint. The patient started with their arm by their side $\left(0^{\circ}\right)$ and was instructed to raise the arm in a sagital plane through $90^{\circ}$. To test the upper limb the start forces were reduced to $10 \mathrm{~N}$ back and forth, a minimum force of $5 \mathrm{~N}$ and acceleration and deceleration set at "medium". For the upper limb isokinetic speeds of 70 and 120 degrees $\cdot \mathrm{s}^{-1}$ were used. Following warm-up and familiarization similar to the lower limb exercise the "overlay" test isolating concentric and eccentric movement was repeated 3 times and the mean peak force for the three movements at both speeds was recorded. 
Acknowledgements: The authors would especially like to thank their respiratory nurses, A. Hay and J. McLean, for their enthusiasm, effort and support of the patients during the training sessions. They are also most grateful to $\mathrm{C}$. Downie and $\mathrm{K}$. Fife and their technical staff for their expertise in evaluating the patients. Thanks also to R. Ewart and R. Hill for the production of the graphs. Finally, they would like to extend special thanks to I. McKay for her help and expertise in preparing the manuscript.

\section{References}

1. American College of Sports Medicine. Guidelines for exercise testing and exercise prescription. 3rd edn. Philadelphia, Lea \& Febiger, 1986.

2. Clark CJ. Setting up a pulmonary rehabilitation programme. Thorax 1994; 49 (3): 270-278.

3. American Thoracic Society. Standards for the diagnosis and care of patients with chronic obstructive pulmonary disease (COPD) and asthma. Am Rev Respir Dis 1986; 136: 225-244.

4. Astrand PO, Rodhall H. In: Textbook of Work Physiology. 2nd edn. New York, McGraw Hill, 1977; pp. 333-365.

5. Jones NL, Campbell EJM. In: Clinical Exercise Testing. 2nd edn. Philadelphia, Saunders, 1981.

6. Gaensler EA, Wright GW. Evaluation of respiratory impairment. Arch Environ Health 1966; 12: 146-189.

7. Spiro SG. Exercise testing in clinical medicine. $\mathrm{Br} \mathrm{J}$ Dis Chest 1977; 71: 145-172.

8. Schonfeld T, Sargent CW, Bautista D, et al. Transcutaneous oxygen monitoring during exercise stress testing. Am Rev Respir Dis 1980; 121: 457-462.

9. Borg G. Perceived exertion as an indicator of somatic stress. Scand J Rehab Med 1970; 2: 92-98.

10. Wasserman K, Hansen JE, Sue EY, Whipp BJ. In: Pathophysiology of Disorders Limiting Exercise: Principles of Exercise Testing and Interpretation. Philadelphia, Lea \& Febiger, 1987; pp. 47-57.
11. Belman MJ. Exercise in patients with chronic obstructive pulmonary disease. Thorax 1993; 48: 936-946.

12. Casaburi R. Exercise training in chronic obstructive lung disease. In: Casaburi R, Petty TL, eds. Principles and Practice of Pulmonary Rehabilitation. Philadelphia, USA, W.B. Saunders Co., 1993; pp. 204-224.

13. Ries AL, Ellis B, Hawkins RW. Upper extremity exercise training in chronic obstructive pulmonary disease. Chest 1988; 93: 688-692.

14. Busch AJ, McClements JD. Effects of a supervised home exercise program on patients with severe chronic obstructive pulmonary disease. Phys Ther 1988; 68 (4): 469-474.

15. Jones DT, Thomson RJ, Sears MR. Physical exercise and resistive breathing training in severe chronic airways obstruction: are they effective? Eur J Respir Dis 1985; 67: 159-166.

16. Astrand PO, Rodhal H. Neuromuscular function. In: Textbook of Work Physiology. 2nd edn. New York, McGraw Hill, 1977; pp. 53-128.

17. Clausen JP. Circulatory adjustments to dynamic exercise and effect of physical training in normal subjects and patients with coronary artery disease. Prog Cardiovasc Dis 1976; 18: 459-495.

18. Ingram RH, Schilder DP. Effect of pursed lips expiration on the pulmonary pressure-flow relationship in obstructive lung disease. Am Rev Respir Dis 1967; 96: 381-388.

19. Casaburi R, Patessio A, Loli F, et al. Reductions in exercise lactic acidosis and ventilation as a result of exercise training in patients with obstructive lung disease. Am Rev Respir Dis 1991; 143: 9-18.

20. Maltais F, LeBlanc P, Simard C, et al. Skeletal muscle adaptation to endurance training in patients with chronic obstructive pulmonary disease. Am J Respir Crit Care Med 1996; 154: 442-447.

21. Taylor R, Jones N. The reduction by training of $\mathrm{CO}_{2}$ output during exercise. Eur J Cardiol 1979; 9 (1): 53-62. 